\title{
Kinetic Parameters during Bis-GMA and TEGDMA Monomer Polymerization by ATR-FTIR: The Influence of Photoinitiator and Light Curing Source
}

\begin{abstract}
Aline B. Denis, Cristina A. Diagone, Ana M. G. Plepis, and Rommel B. Viana
Instituto de Química de São Carlos, Universidade de São Paulo, Avenida Trabalhador São Carlense, No. 400, Caixa Postal 780, Bairro: Centro, 13560-970 São Carlos, SP, Brazil

Correspondence should be addressed to Rommel B. Viana; rommelbv@yahoo.com.br

Received 10 January 2016; Revised 23 May 2016; Accepted 15 June 2016

Academic Editor: Austin Nevin

Copyright (C) 2016 Aline B. Denis et al. This is an open access article distributed under the Creative Commons Attribution License, which permits unrestricted use, distribution, and reproduction in any medium, provided the original work is properly cited.

This study aimed to analyze the kinetic parameters of two monomers using attenuated total reflectance Fourier transform infrared (ATR-FTIR): 2,2-bis-[4-(2-hydroxy-3-methacryloxypropyl-1-oxy)-phenyl] propane (Bis-GMA) and triethylene glycol dimethacrylate (TEGDMA). The following were calculated to evaluate the kinetic parameters: maximum conversion rate $\left(\mathrm{Rp}^{\max }\right)$, time at the maximum polymerization rate $\left(t_{\max }\right)$, conversion at $\mathrm{Rp}^{\max }$, and total conversion recorded at the maximum conversion point after $300 \mathrm{~s}$. Camphorquinone (CQ) and phenyl propanedione (PPD) were used in this study as photoinitiators, whereas N,Ndimethyl-p-toluidine (DMPT) amine was used as a coinitiator. LED apparatus and halogen lamp were used in turn to evaluate the effect that light source had on the monomer kinetics. The mass concentration ratio for the three resin preparations was $0.7: 0.3$ for Bis-GMA and TEGDMA: R1 (CQ + DMPT), R2 (PPD + DMPT), and R3 (PPD + CQ + DMPT). The PPD association with the CQ photoinitiator altered the polymerization kinetics compared to a resin containing only the CQ photoinitiator. The light sources exhibited no significant differences for $t_{\max }$ of R1 and R3. Resins containing only the PPD initiator exhibited a higher $t_{\max }$ than those containing only CQ. However, the $\mathrm{Rp}^{\max }$ decreased for resins containing the PPD photoinitiator.
\end{abstract}

\section{Introduction}

The emergence of composite resins with optimal adhesion to enamel and dentin walls greatly changed dental restoration because repairs could be performed without causing wear to a healthy tooth. For over fifty years, resinous materials have been intensively used in odontological clinics, although such materials have changed often to improve their physicomechanical, biological, and esthetic properties [1].

Higher degrees of conversion (DC) provide restoration materials with better mechanical properties, such as wear, compressive, shear, and flexural strength and hardness [26]. However, a high DC internally generates contraction stresses in the material that are partially transmitted to the tooth-restoration adhesive interface. This compromises the marginal integrity and forms gaps that may allow microinfiltration of the restoration and postoperative sensitivity [7, 8]. However, insufficient polymerization of the composite resin interferes with not only the mechanical properties of the material but also its biological properties, preferentially liberating components not bonded to the polymer network and possibly causing allergic reactions and both cytotoxic and genotoxic effects. Additionally, these residual monomers may reach the dental pulp via dentinal tubules [9-12]. Thus, better material polymerizations improve their biocompatibility, which is associated with the nature and quantity of released components [13].

The monomer DC for resins is influenced by the monomer composition, photoinitiators, energy density, and wavelength for the photoactivation technique used to initiate the polymerization process [14-16]. Currently, the most used photoinitiator for odontological resinous materials is camphorquinone (CQ), although it has some disadvantages, such as its intense yellow color, which affects the incorporation of the brightly colored resins that are widely used in whitened teeth. Additionally, it exhibits a low polymerization efficiency [17]. Other photoinitiators, such as phenyl propanedione (PPD), which is less dependent on tertiary amines to generate 
free radicals, are being tested to bypass such problems. In addition to its bright yellow color, PPD is a liquid at room temperature, which aids in incorporating higher quantities into composite resins [17-20].

Studies are being conducted to evaluate the association of CQ with PPD in the same resin $[18,20]$ because the contraction stresses in the resin may reduce by decreasing the maximum polymerization rate $\left(\mathrm{Rp}^{\mathrm{max}}\right)$ without changing the DC [20-22]. Such brighter photoinitiators absorb light closer to the ultraviolet region than CQ [19]. This wavelength is covered perfectly by a halogen lamp, although it is not completely provided by most LED equipment because of their narrow emission spectrum of $470 \pm 20 \mathrm{~nm}$. This difference causes resins containing these photoinitiators to polymerize poorly when using such equipment, which potentially yields a low DC and inferior mechanical properties [19, 23]. However, associating the two photoinitiators (CQ and PPD) yields a maximum absorption peak at approximately $452 \mathrm{~nm}$ [20], which is perfect for activation by equipment within the blue band of the electromagnetic spectrum. Furthermore, LEDs are increasingly used in odontological clinics, with a tendency to completely substitute traditional halogen lamp photoactivation equipment due to the advantage of such equipment. Therefore, the effectiveness of such equipment for resins using PPD photoinitiators must be studied.

Studies evaluating the polymerization kinetics of composite resins containing other photoinitiators such as PPD are still required, and little is known about residual monomers that can be liberated from such composite resins. Real-time Fourier transform infrared (RT-FTIR) spectroscopy is a nondestructive technique widely used in recent years to follow reaction kinetics of such photoactivated composite resins [24]. This technique has been used in studies to evaluate the monomer DC and composite resin polymerization kinetics [25-29].

This study aimed to evaluate the DC and polymerization kinetic parameters for experimental resins using different photoinitiators to evaluate the kinetic parameters for residual monomers 2,2-bis- [4-(2-hydroxy-3-methacryloxypropyl-1-oxy)-phenyl] propane (Bis-GMA) and triethylene glycol dimethacrylate (TEGDMA) in experimental resins containing different photoinitiators. The photoactivator equipment performance on the experimental resins will also be tested, as will the hypothesis that experimental resin performance will be higher or equal using two photoinitiators (CQ + PPD) than just CQ, as based on the DC results. Additionally, the hypothesis that experimental resins with two photoinitiators $(\mathrm{CQ}+\mathrm{PPD})$ exhibit a reduced maximum polymerization rate $\left(\mathrm{Rp}^{\mathrm{max}}\right)$ relative to ones containing only CQ will be tested. The hypothesis that the LED apparatus will perform similarly to the halogen lamp for experimental resins with both photoinitiators (CQ + PPD) will also be examined.

\section{Materials and Methods}

The dimethacrylate monomers 2,2-bis-[4-(2-hydroxy-3-met hacryloxypropyl-1-oxy)-phenyl] propane (Bis-GMA/Aldrich, batch $06627 \mathrm{BH}$ ) and triethylene glycol dimethacrylate
(TEGDMA/Aldrich, batch 07709KE437) were used to manipulate the experimental resins. The substances used to photoinitiate the organic matrix were $\mathrm{N}, \mathrm{N}$-dimethylp-toluidine amine (DMTP; Fluka, batch 32107361) as the coinitiator and camphorquinone (CQ; dl-2,3-diketo1,7,7-trimethyl norcamphane, Fluka, batch 1293239) and phenyl propanedione (PPD; Aldrich, batch photoinitiators S34870-116) as the photoinitiators.

Three experimental resins were prepared by combining Bis-GMA and TEDGMA monomers with a mass concentration ratio of $0.7: 0.3$ and by incorporating the photoinitiation systems (see below). The photoinitiator to coinitiator weight ratio was held constant $(1: 1)$ for all resins. Resin R1 refers to a mixture of CQ $(0.2 \%$ in weight) with DMPT ( $0.2 \%$ in weight), $\mathrm{R} 2$ is a mixture of PPD ( $0.2 \%$ in weight) with DMPT $(0.2 \%$ in weight), and R3 is a mixture of PPD ( $0.1 \%$ in weight $)+C Q$ ( $0.1 \%$ in weight) with DMPT ( $0.2 \%$ in weight).

The following photoactivation apparatuses were used: (a) a Demetron LC/SDS Halogen lamp Kerr (sn- 67009594, USA) and (b) an LED Poly 600/Kavo (sn- 2008100188, Brazil). These photoactivation pieces of equipment were used with a $600 \mathrm{~mW} \mathrm{~cm}^{-2}$ power density for 40 seconds on each specimen. The two equipment pointers were cylindrical and $10 \mathrm{~mm}$ in diameter, whereas the energy dosage for both was $24 \mathrm{~J} \mathrm{~cm}^{-2}$. The light intensity or power density for the apparatuses was measured and standardized using a potentiometer (Fieldmaster/Coherent).

The DC and polymerization kinetics for the experimental resins were determined using a Fourier transform infrared spectrophotometer (NEXUS 670 FT-IR/Nicolet, nsAEQ0000517). This equipment contains an attenuated total reflectance (ATR) unit corresponding to a platform coupled to the spectrophotometer with a set of mirrors that directs light into the Zinc Selenide (ZnSe) crystal, which is located on the platform surface and works as an active substrate for the infrared irradiation.

A Teflon matrix was placed on the crystal surface, the experimental resins were inserted into the matrix orifice ( $5 \mathrm{~mm}$ in diameter by $1 \mathrm{~mm}$ deep), and a polyester strip was placed over the resin at the top of the matrix with close contact to the photoactivation source. The infrared spectra for these samples were collected in the kinetics mode at three scans per second for 305 seconds with a $2 \mathrm{~cm}^{-1}$ resolution from 1680 to $1550 \mathrm{~cm}^{-1}$. The first five scans collected provided the absorption spectrum for the nonpolymerized resin; by the sixth scan, the photoactivator had been activated for only 40 seconds, and the spectrum had been collected for 300 seconds after the initial light activation. Five repetitions were performed for each experimental condition, and all analyses were conducted at a controlled temperature $\left(23 \pm 1^{\circ} \mathrm{C}\right)$ and humidity $(60 \pm 5 \%)$.

The percent nonconverted double carbon bonds $(\% \mathrm{C}=\mathrm{C})$ were determined using the absorbance peak intensities for the $\mathrm{C}=\mathrm{C}$ bonds at $1637 \mathrm{~cm}^{-1}$ (peak corresponding to the aliphatic chains) and $\mathrm{C}=\mathrm{C}$ bonds at $1610 \mathrm{~cm}^{-1}$ (peak corresponding to the aromatic chains) before and after the polymerization (referring to (1)) $[5,6,27,28]$. The corresponding DC was calculated by subtracting this rate from $100 \%$ : 


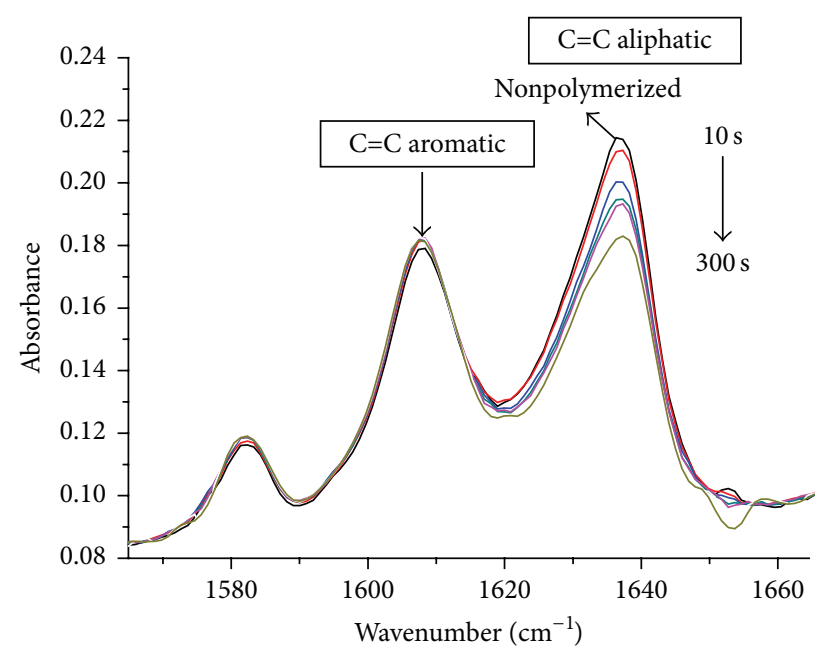

(a)

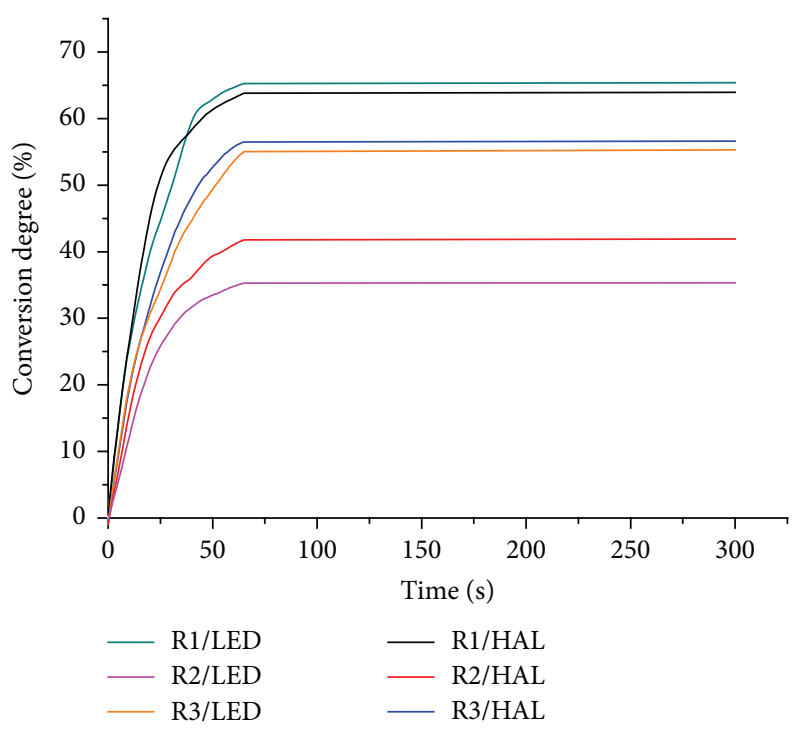

(b)

FIGURE 1: (a) Real-time FT-IR spectrum of the carbon double bond conversion for the peak corresponding to the aliphatic chains. (b) Graph with the real-time DC for resins using LED and halogen lamp photoactivation.

$$
\begin{aligned}
& (\% \mathrm{C}=\mathrm{C}) \\
& =\frac{\operatorname{Abs}\left(1.637 \mathrm{~cm}^{-1}\right) / \operatorname{Abs}\left(1.610 \mathrm{~cm}^{-1}\right) \text { polymer }}{\operatorname{Abs}\left(1.637 \mathrm{~cm}^{-1}\right) / \operatorname{Abs}\left(1.610 \mathrm{~cm}^{-1}\right) \text { monomer }} \\
& \mathrm{DC}=100 \%-(\% \mathrm{C}=\mathrm{C}) \text {. }
\end{aligned}
$$

The calculated polymerization kinetic parameters were the maximum conversion rate $\left(\mathrm{Rp}^{\max }\right)$, the time when the $\mathrm{Rp}^{\max }\left(t_{\max }\right)$ occurred, the conversion at this time, and the total conversion registered at the maximum conversion point, that is, after $300 \mathrm{~s}$. The conversion rate (Rp) was calculated from the first derivative of the real-time conversion profiles; therefore, the highest polymerization rate could be calculated at an interval of 1 second $\left(\mathrm{Rp}^{\mathrm{max}}\right)$. The other parameters were calculated from the real-time conversion values and conversion rate. The Kolmogorov-Smirnov test was used to verify that these data were normally distributed [30]. None of the groups deviated significantly from normality $(p>0.05)$; hence, parametric tests were used for the statistical analyses. Analysis of variance (ANOVA) with two criteria and Tukey's post hoc test were used to compare the resins (R1, R2, and R3) and photoactivation sources (halogen lamp and LED). A significance level of 5\% $(p<0.05)$ was adopted for all tests. All of the statistical procedures were executed using Statistica version 5.1 (StatSoft Inc., Tulsa, USA).

\section{Results and Discussion}

The FT-IR spectra for the monomer conversion degree in the resins based on the photoactivation time are shown in Figure 1(a). The carbon double bond peak conversion to the corresponding aliphatic chains began with the resin photoactivation, especially during the first few seconds, and was observed in real-time to 300 seconds as can be seen in Figure 1(b). Furthermore, Figure 2 shows the monomer conversion degree for the three resins (R1, R2, and R3) photoactivated by the 2 different light sources (LED and halogen lamp) after 300 seconds. A statistical analysis was performed to verify the significance of the numeric differences between these groups. A parametric statistical test was selected, ANOVA with two criteria, for the two variables (the resin and light source). Tukey's post hoc test at a significance level of $5 \%(p<0.05)$ was also used for multiple comparisons and iterations

The statistical results (referring to Table S1 in Supplementary Material available online at http://dx.doi.org/10 $.1155 / 2016 / 6524901)$ indicated that all of the resins were significantly different and that only resin R2 exhibited a significant difference between the light sources, whereas the other resins were not significantly different. The lowest average conversion degree occurred when resin R2 was photoactivated by the LED $(35.64 \% \pm 1.73)$, and the highest conversion occurred for resin R1 photoactivated by both the LED $(65.90 \% \pm 1.81)$ and the halogen lamp $(64.40 \% \pm 0.83)$.

The real-time analysis of the resin conversion using ATR-FTIR for the three resins photoactivated by the two light sources indicated that most of the reaction occurred during the first few seconds starting once the photoactivation equipment was activated (see Figure 1(b)). The reaction occurred especially rapidly during the first 60 seconds, and no significant alterations occurred after this period. The maximum conversion rates $\left(\mathrm{Rp}^{\max }\right)$ are shown in Figure 3. Because of variations in the values, a statistical analysis of the $\mathrm{Rp}^{\max }$ results was performed and indicated that all of the resins were significantly different, with no difference between the light sources for the same resin (referring to Table S2). The highest average $\mathrm{Rp}^{\max }$ was detected for resin 


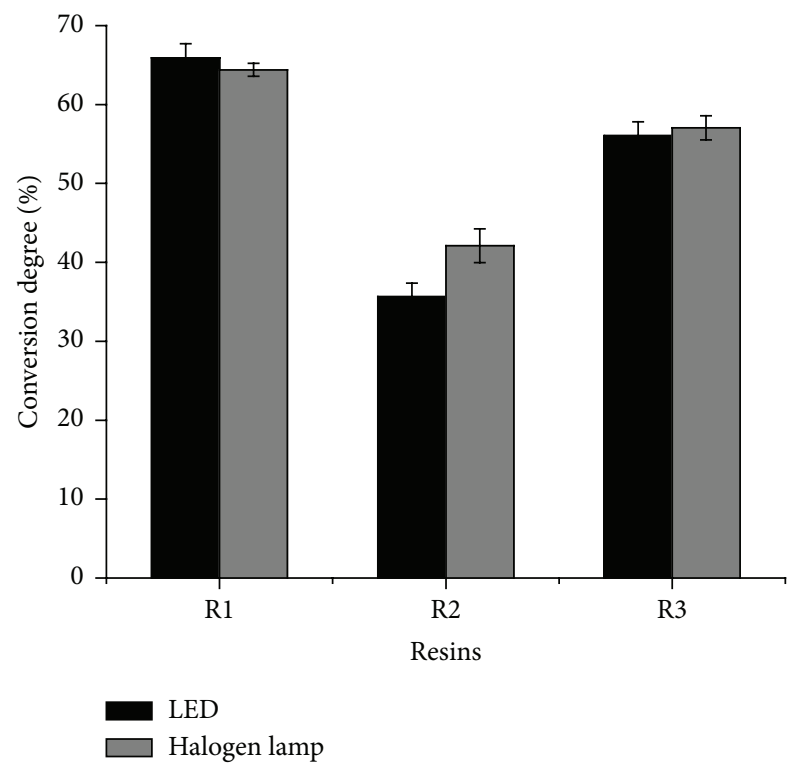

FIGURE 2: Graph of the mean and standard deviation for the DC (\%) after 300 seconds for the three resins (R1, R2, and R3) photoactivated by two light sources (LED and halogen lamp).

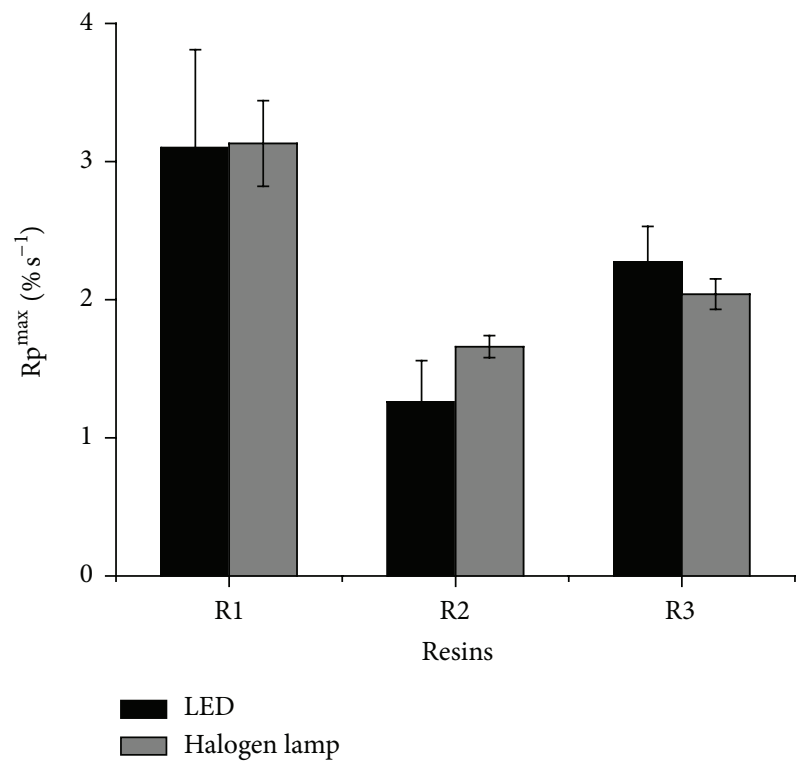

FIGURE 3: Graph of the mean and standard deviation for $\mathrm{Rp}^{\max }$ for the three resins (R1, R2, and R3) photoactivated by two light sources (LED and halogen lamp).

$\mathrm{R} 1\left(3.13 \pm 0.31 \% \mathrm{~s}^{-1}\right)$ photoactivated by the halogen lamp, and the lowest average value was from resin $\mathrm{R} 2(1.26 \pm$ $0.30 \% \mathrm{~s}^{-1}$ ) photoactivated by the LED. The lowest $\mathrm{Rp}^{\max }$ values were given by resins (R2 and R3) containing the $\mathrm{PPD}$ photoinitiator in their composition versus the resin containing only CQ (R1).

The $t_{\max }$ values are shown in Figure 4 . The maximum conversion rate varied from 4 to 10 seconds. The two light sources exhibited no significant influence on the $t_{\max }$ values for resins

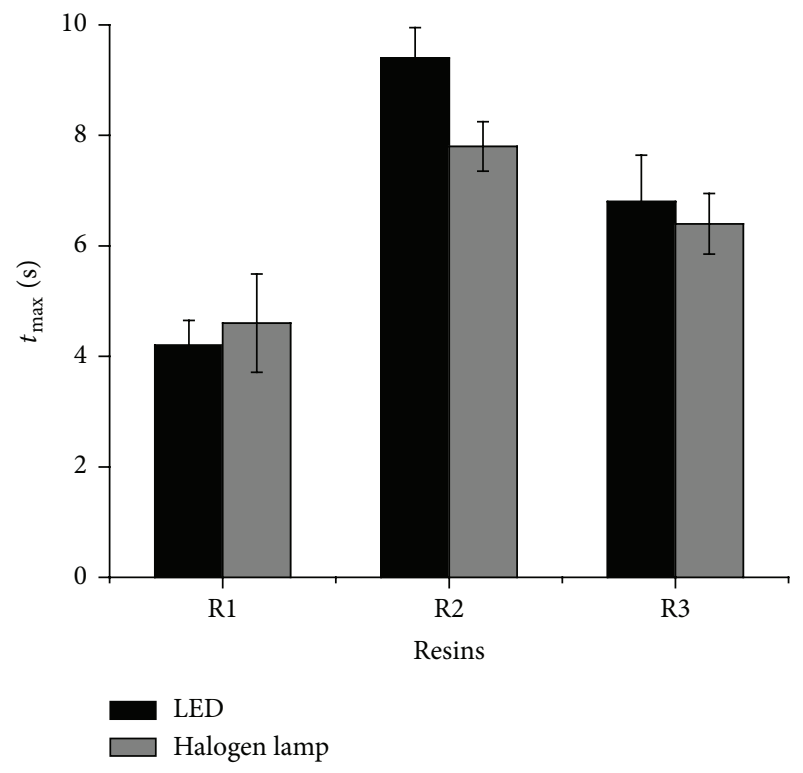

FIGURE 4: Graph of the mean and standard deviation for the $t_{\max }$ for the three resins (R1, R2, and R3) photoactivated by two light sources (LED and halogen lamp).

R1 and R3 (referring to Tables S3 and S4). However, when photoactivated by the LED, resin R2 differed significantly relative to all other groups, with the highest average $t_{\max }$ $(9.40 \pm 0.55 \mathrm{~s})$. The resins containing PPD yielded $t_{\max }$ values above those containing only CQ. The conversion degree at the maximum conversion rate $\left(t_{\max }\right)$ ranged from $8.75 \%$ for resin R2 photoactivated by the LED to $17.04 \%$ for resin R1 photoactivated by the halogen lamp.

This study demonstrated that associating PPD with CQ altered the polymerization reaction kinetics for resins relative to only using CQ. The resin DC is normally 45 to $75 \%$, and the polymerization reaction is never complete, which leaves unconverted monomers [4, 31, 32]. However, the minimum DC for clinical resin applications are not well established. This incomplete polymerization reaction occurs because the BisGMA monomer contains aromatic groups in the center of its chain, which prevents the two acrylic groups at the chain ends from moving freely [33]. Because of this rigidity, the monomer becomes viscous and requires a comonomer, which is generally triethylene glycol dimethacrylate (TEGDMA), to adjust the viscosity. Thus, only one methacrylate group is active while the other becomes less active and only occasionally cross polymerizes with adjacent molecules, which decreases the DC. The viscosity of a Bis-GMA/TEGDMA mixture should generally be between 1 and $2 \mathrm{~Pa}$, which corresponds to a weight ratio between $0.5: 0.5$ and $0.7: 0.3$, respectively $[13,34-36]$. Some studies have demonstrated that incorporating smaller quantities of the TEGDMA diluent monomer versus Bis-GMA provides superior mechanical properties and lowers the polymerization contraction values $[35,36]$, which justifies the Bis-GMA/TEGDMA ratio used in this study.

The total DC after 300 seconds for resin R3 (which combines PPD with CQ as photoinitiators) yielded intermediary 
values relative to the other resins. The highest conversion values were exhibited by R1 photoactivated by either the LED or the halogen lamp, whereas the lowest values were found for R2 photoactivated by the LED. Notably, R2 was the only resin that exhibited a significant difference between the light sources. These different DC for the two light sources occurred for R2 because of the different wavelengths for this equipment. While the LED emits wavelengths from 440 to $480 \mathrm{~nm}$, the halogen lamp emits from 400 to $525 \mathrm{~nm}$. The LED device provided the worst overall performance when photoactivating resin R2 (PPD as photoinitiator); however, when used for resin R1 (which only contains CQ), it provided the best overall performance, and comparable values for the halogen lamp were obtained for R3 (CQ + PPD).

According to Neumann et al. [19], the PPD wavelength is perfectly covered by the halogen lamp but is incompletely activated by most LED equipment because of its narrow emission spectrum. This trait indicates that such equipment inadequately photoinitiates such resins, which can yield low DC and inferior mechanical properties [16, 19, 23]. Some authors believe this lower efficiency is caused not only by the light absorption characteristics of the photoinitiator but also by its photochemical characteristics [22, 37, 38]. Furthermore, combining the two photoinitiators (CQ + PPD) produces a maximum light absorption peak at approximately $452 \mathrm{~nm}$ [20], which is perfect for activation by equipment in the blue range of the electromagnetic spectrum, as observed in this study. However, recent studies, such as that by Brandt et al. [39], found that resins containing PPD photoinitiator obtained a lower DC when photoactivated by halogen lamps. Brandt et al. [39] also detected a DC similar to that of R1 photoactivated by LED equipment, and the authors suggest that this effectiveness occurred because of the high energy dosages $\left(35.5 \mathrm{~J} \mathrm{~cm}^{-2}\right)$ provided by third-generation LED equipment such as Ultralume LED5/Ultradent $\left(1.315 \mathrm{~mW} \mathrm{~cm}^{-2}\right)$. Notably, a similar DC is obtained using the light source distribution spectrum, which is wider for such equipment but also matches the light absorption capability of the photoinitiator. Additionally, power intensity also influences the kinetics of the reaction and final polymer properties when comparing CQ and PPD, as demonstrated by Schneider et al. [20].

Some studies report better DC for resins containing only PPD than for resins with CQ $[5,13,17,18]$. According to Park et al. [18] and Sun and Chae [17], there may be a synergic effect to combining CQ + PPD due to a second form of free radical generation via the abstraction of photons and photocleavage. Furthermore, this combination could yield a wider absorption spectrum and increase photoinitiator system efficiency [17, 18]; however, Neumann et al. [19] did not detect such a synergic effect. This study indicated an intermediate DC for resins containing the two photoinitiators CQ + PPD, as also observed by Schneider et al. [21] when the photoinitiators were used at low concentrations with a $1: 1$ ratio (photoinitiator: amine). Furthermore, Schneider et al. [21] and Brandt et al. [39] did not find a significant difference between resins containing CQ + PPD and those with only CQ. Some studies performed using the PPD photoinitiator suggest that a tertiary amine is not required for the polymerization reaction because it is a type I photoinitiator that generates free radicals via fragmentation (photocleavage); therefore, PPD is less dependent on the coinitiator to generate free radicals [17-19]. However, more recent studies demonstrate that PPD performs better with a coinitiator; therefore, there is still some doubt regarding the free radical formation mechanisms for these photoinitiators [14, 22, 36, 37].

The rate dependence on amine (coinitiator) has also been studied. Different quantities of amine relative to the photoinitiator yield different results. Values from $0.1 \%$ to $3.5 \%$ in weight were studied [13, 18-39]. However, high photoinitiator concentrations may compromise the final resin aesthetics, especially for those with brighter colors. Furthermore, the increase in amine concentration in the photoinitiation system is limited because of its cytotoxic, carcinogenic, and mutagenic characteristics [40]. Excessive amine can delay the reaction process and contribute to the initial yellowing of the polymer and subsequent darkening of the resin duet to the rapid oxidation of these compounds [41]. Park et al. [18] studied different photoinitiators quantities between 0 and $3.2 \%$ in weight and a mixture of the two agents with a limit of $3.4 \%$ in weight and found that the highest DC occurred by combining PPD with CQ in a $1: 1$ to $1: 4$ ratio. Notably, higher PPD concentrations increased the DC, which did not occur with CQ, and improved with high concentrations. Schneider et al. [21] also studied the rates for different photoinitiators and amines for CQ and PPD using ratios of $2: 1,1: 1,1: 15$, and $1: 2$, with $0.3 \%$ photoinitiator weight and an amine concentration from $0.15 \%$ to $0.6 \%$ in weight. Schneider et al. [21] determined that ratios of $1: 15$ and $1: 2$ yielded the best results, regardless of the photoinitiator. However, according to Peutzfeldt and Asmussen [42], CQ and amine concentrations of $0.1 \%$ to $0.2 \%$ are the most acceptable, and this is notably caused by not only the photoinitiator quantity but also by the type of amine.

Some studies $[17-19,43]$ indicate PPD may cause less yellowing of the resin (in relation to CQ) because PPD is more brightly colored than $\mathrm{CQ}$, which makes incorporating it into the resin in larger amounts easier. However, Schneider et al. [20] proved that this decreased degree of resin yellowing only occurred when PPD was used at low concentrations $(0.33 \%)$. Additionally, some authors believe that the polymerization reaction with PPD is slower [14, 20,39], which decreases the maximum conversion rate $\left(\mathrm{Rp}^{\max }\right)$ without altering the values DC relative to CQ $[5,14,20,39]$.

This study found that the $\mathrm{Rp}^{\max }$ decreased in resins containing the PPD photoinitiator. The highest $\mathrm{Rp}^{\max }$ was found for R1 $(3.13 \% / \mathrm{s} \pm 0.31)$ photoactivated by the halogen lamp, while the lowest value was found for R2 $(1.26 \% / \mathrm{s} \pm 0.30)$ photoactivated by the LED. However, significant differences in DC were found between the three resins. No synergic effect between CQ + PPD was detected in this study in contrast to that detected by Park et al. [18], who observed higher conversions with this association. An explanation for this difference could be the high photoinitiator concentrations used by these authors (1.8\% in weight with a $1: 1$ ratio) [18]. In contrast, Neumann et al. [44, 45] observed lower conversions 
for the PPD and CQ photoinitiators, which suggests a possible reaction between PPD and the resinous matrix, which altered the absorption values and could have been caused by an altered conformation in the carbonyl groups. Therefore, the first hypothesis for this study is incorrect because the resin with both photoinitiators (CQ + PPD) yielded a lower DC than the resin containing only CQ. In contrast, the second hypothesis for this study is correct because the resin exhibited a better maximum polymerization rate $\left(\mathrm{Rp}^{\mathrm{max}}\right)$, that is, a lower $\left(\mathrm{Rp}^{\max }\right)$. According to Schneider et al. [22] the reaction slows because of the reduced maximum polymerization rate $\left(\mathrm{Rp}^{\mathrm{max}}\right)$ promoted by the PPD photoinitiator, which reduces the maximum stress rate and, consequently, decreases the formation of gaps that cause microinfiltration of the restoration and postoperatory sensitivity [1].

The maximum conversion rate $\left(t_{\max }\right)$ occurred sooner for the resin containing only the CQ initiator (R1). In contrast, adding PPD caused the $t_{\max }$ to occur later. The time for the maximum conversion rate varied from $4.20 \mathrm{~s} \pm 0.45 \mathrm{~s}$ to $9.40 \mathrm{~s}$ $\pm 0.55 \mathrm{~s}$, which is similar to the results of Schneider et al. [20]. Additionally, a slight difference in $t_{\max }$ was found within the group itself and may be due to the manual triggering of the photoactivator, causing slight differences in the activation timing, which changes $t_{\max }$. Schneider et al. [21] believed that resins with PPD exhibited a later $t_{\max }$ because the PPD system is less reactive due to its lower interactions with the coinitiator.

Based on $t_{\max }$ results, resin photoactivation protocols that recommend a reduced initial exposure time may affect the DC of the resin and, consequently, its properties. Denis et al. [27] decreased $t_{\max }$ by modulating the photoactivation source: that is, the light intensity of the photoactivation equipment starts being lower and increases over time. This decreased light intensity is believed to better accommodate the molecules initially, which decreases the contraction stress in the resin because of the slower conversion [46]. In turn, some authors believe an initial slow polymerization provides few polymer formation centers, which yields a more linear structure with a low reticulation degree and may increase the wear and degradation of the resin in the buccal medium and is still a controversial subject [47-50].

All of the resins prepared in this study yielded a similar DC at $t_{\max }$, that is, at the time of the maximum conversion rate. These values varied between $11.90 \%$ and $15.33 \%$, indicating that the photoinitiator did not influence the DC at $\mathrm{Rp}^{\max }$. Notably, after $t_{\max }$, the increase of viscosity and the reduced free volume restricted progressively the polymeric chain mobility, which limited the reaction propagation.

\section{Conclusions}

This study aimed to evaluate the polymerization kinetic parameters for Bis-GMA and TEGFMA monomers. The effects of the photoinitiator (CQ and PPD) and different light sources (LED, halogen lamp) on the polymerization kinetic parameters were considered. (i) The first hypothesis evaluated by this study was that an experimental resin using two photoinitiators (CQ + PPD) would perform better or equal to a resin containing only CQ based on the DC.
However, the results of this study indicate that this hypothesis is incorrect because the resin with two photoinitiators (CQ + PPD) exhibited a lower DC than the resin containing only CQ. (ii) Additionally, the hypothesis that the experimental resin with two photoinitiators (CQ + PPD) would exhibit a reduced maximum polymerization rate $\left(\mathrm{Rp}^{\mathrm{max}}\right)$ relative to one containing only CQ was also investigated. In this case, the hypothesis was correct, and using both photoinitiators $(\mathrm{CQ}+\mathrm{PPD})$ in the same resin improved the maximum polymerization rate $\left(\mathrm{Rp}^{\max }\right)$; that is, $\mathrm{Rp}^{\max }$ was smaller than for the resin containing only CQ. (iii) Another aspect examined in this study was the hypothesis that the LED equipment performs similarly to the halogen lamp for the experimental resin with both photoinitiators (CQ + PPD). This hypothesis was also correct because the LED performed similarly to the halogen lamp for the resin containing both photoinitiators (CQ + PPD), which proves its effectiveness for this use.

\section{Competing Interests}

The authors declare that they have no competing interests.

\section{Acknowledgments}

The authors thank the National Council for Scientific and Technological Development [Conselho Nacional de Desenvolvimento Científico e Tecnológico (CNPq)], the São Paulo Research Foundation [Fundação de Amparo à Pesquisa do Estado de São Paulo (FAPESP)], and the Brazilian Federal Agency for the Support and Evaluation of Graduate Education [Coordenação de Aperfeiçoamento de Pessoal de Nível Superior (CAPES)] for their financial support. Rommel B. Viana also thanks CAPES for the research fellowship.

\section{References}

[1] J. L. Ferracane, "Resin composite-state of the art," Dental Materials, vol. 27, no. 1, pp. 29-38, 2011.

[2] J. L. Ferracane, "Correlation between hardness and degree of conversion during the setting reaction of unfilled dental restorative resins," Dental Materials, vol. 1, no. 1, pp. 11-14, 1985.

[3] A. Peutzfeldt, "Resin composites in dentistry: the monomer systems," European Journal of Oral Sciences, vol. 105, no. 2, pp. 97-116, 1997.

[4] A. Peutzfeldt and E. Asmussen, "Resin composite properties and energy density of light cure," Journal of Dental Research, vol. 84, no. 7, pp. 659-662, 2005.

[5] E. Asmussen and A. Peutzfeldt, "Influence of composition on rate of polymerization contraction of light-curing resin composites," Acta Odontologica Scandinavica, vol. 60, no. 3, pp. 146-150, 2002.

[6] E. Asmussen and A. Peutzfeldt, "Influence of selected components on crosslink density in polymer structures," European Journal of Oral Sciences, vol. 109, no. 4, pp. 282-285, 2001.

[7] N. Hofmann, W. Denner, B. Hugo, and B. Klaiber, "The influence of plasma arc vs. halogen standard or soft-start irradiation on polymerization shrinkage kinetics of polymer matrix composites," Journal of Dentistry, vol. 31, no. 6, pp. 383393, 2003. 
[8] M. Atai and D. C. Watts, "A new kinetic model for the photopolymerization shrinkage-strain of dental composites and resin-monomers," Dental Materials, vol. 22, no. 8, pp. 785-791, 2006.

[9] S. Schwengberg, H. Bohlen, N. Kleinsasser et al., "In vitro embryotoxicity assessment with dental restorative materials," Journal of Dentistry, vol. 33, no. 1, pp. 49-55, 2005.

[10] J. C. Wataha, F. A. Rueggeberg, C. A. Lapp et al., "In vitro cytotoxicity of resin-containing restorative materials after aging in artificial saliva," Clinical Oral Investigations, vol. 3, no. 3, pp. 144-149, 1999.

[11] N. H. Campanha, A. C. Pavarina, E. T. Giampaolo, A. L. Machado, I. Z. Carlos, and C. E. Vergani, "Cytotoxicity of hard chairside reline resins: effect of microwave irradiation and water bath postpolymerization treatments," International Journal of Prosthodontics, vol. 19, no. 2, pp. 195-201, 2006.

[12] M. Goldbery, "In vitro and in vivo studies on the toxicity of dental resin components: a review," Clinical Oral Investigations, vol. 12, no. 1, pp. 1-8, 2008.

[13] R. E. López Palacio and C. A. De Carvalho Zavaglia, “The monomeric formulation optimization of dental composite: mechanical and kinetic studies," Artificial Organs, vol. 27, no. 5, pp. 419-423, 2003.

[14] N. Emami and K. M. Söderholm, "Influence of light-curing procedures and photo-initiator/co-initiator composition on the degree of conversion of light-curing resins," Journal of Materials Science: Materials in Medicine, vol. 16, no. 1, pp. 47-52, 2005.

[15] P. H. P. D’Alpino, N. R. Svizero, J. C. Pereira, F. A. Rueggeberg, R. M. Carvalho, and D. H. Pashley, "Influence of light-curing sources on polymerization reaction kinetics of a restorative system," American Journal of Dentistry, vol. 20, no. 1, pp. 46-52, 2007.

[16] A. Ogunyinka, W. M. Palin, A. C. Shortall, and P. M. Marquis, "Photoinitiation chemistry affects light transmission and degree of conversion of curing experimental dental resin composites," Dental Materials, vol. 23, no. 7, pp. 807-813, 2007.

[17] G. J. Sun and K. H. Chae, "Properties of 2,3-butanedione and 1-phenyl-1,2-propanedione as new photosensitizers for visible light cured dental resin composites," Polymer, vol. 41, no. 16, pp. 6205-6212, 2000.

[18] Y. J. Park, K. H. Chae, and H. R. Rawls, "Development of a new photoinitiation system for dental light-cure composite resins," Dental Materials, vol. 15, no. 2, pp. 120-127, 1999.

[19] M. G. Neumann, C. C. Schmitt, G. C. Ferreira, and I. C. Corrêa, "The initiating radical yields and the efficiency of polymerization for various dental photoinitiators excited by different light curing units," Dental Materials, vol. 22, no. 6, pp. 576-584, 2006.

[20] L. F. J. Schneider, C. S. C. Pfeifer, S. Consani, S. A. Prahl, and J. L. Ferracane, "Influence of photoinitiator type on the rate of polymerization, degree of conversion, hardness and yellowing of dental resin composites," Dental Materials, vol. 24, no. 9, pp. 1169-1177, 2008.

[21] L. F. J. Schneider, L. M. Cavalcante, S. Consani, and J. L. Ferracane, "Effect of co-initiator ratio on the polymer properties of experimental resin composites formulated with camphorquinone and phenyl-propanedione," Dental Materials, vol. 25, no. 3, pp. 369-375, 2009.

[22] L. F. J. Schneider, S. Consani, R. L. Sakaguchi, and J. L. Ferracane, "Alternative photoinitiator system reduces the rate of stress development without compromising the final properties of the dental composite," Dental Materials, vol. 25, no. 5, pp. 566-572, 2009.

[23] A. Uhl, R. W. Mills, and K. D. Jandt, "Photoinitiator dependent composite depth of cure and Knoop hardness with halogen and LED light curing units," Biomaterials, vol. 24, no. 10, pp. 1787$1795,2003$.

[24] M. Daronch, F. A. Rueggeberg, M. F. De Goes, and R. Giudici, "Polymerization kinetics of pre-heated composite," Journal of Dental Research, vol. 85, no. 1, pp. 38-43, 2006.

[25] R. R. Braga and J. L. Ferracane, "Contraction stress related to degree of conversion and reaction kinetics," Journal of Dental Research, vol. 81, no. 2, pp. 114-118, 2002.

[26] M. Trujillo, S. M. Newman, and J. W. Stansbury, "Use of nearIR to monitor the influence of external heating on dental composite photopolymerization," Dental Materials, vol. 20, no. 8, pp. 766-777, 2004.

[27] A. B. Denis, R. B. Viana, and A. M. G. Plepis, "Kinetic parameters and monomeric conversion of different dental composites using standard and soft-start photoactivation modes," Laser Physics, vol. 22, no. 6, pp. 1099-1104, 2012.

[28] A. B. Denis, A. M. G. Plepis, and R. B. Viana, "The effect of different photoactivation sources on a nanocomposite resin," Acta Scientiarum Technology, vol. 35, pp. 407-412, 2013.

[29] A. B. Denis, A. M. G. Plepis, V. D. C. A. Martins, J. C. Pereira, R. Cilli, and A. Prakki, "Properties of experimental resins based on synthesized propoxylated bis-GMA with different propionaldehyde ratios," Materials Research, vol. 15, no. 3, pp. 397-402, 2012.

[30] N. M. Razali and Y. B. Wah, "Power comparations of ShapiroWilk, Kolmogorov-Smirnov, Lilliefors and Anderson-Darling tests," Journal of Statistical Modeling and Analytics, vol. 2, pp. 21-33, 2011.

[31] J. L. Ferracane and J. R. Condon, "Rate of elution of leachable components from composite," Dental Materials, vol. 6, no. 4, pp. 282-287, 1990.

[32] J. L. Ferracane, "Elution of leachable components from composites," Journal of Oral Rehabilitation, vol. 21, no. 4, pp. 441-452, 1994.

[33] E. Andrzejewska, "Photopolymerization kinetics of multifunctional monomers," Progress in Polymer Science, vol. 26, no. 4, pp. 60-65, 2001.

[34] I. D. Sideridou and M. M. Karabela, "Sorption of water, ethanol or ethanol/water solutions by light-cured dental dimethacrylate resins," Dental Materials, vol. 27, no. 10, pp. 1003-1010, 2011.

[35] I. Sideridou, V. Tserki, and G. Papanastasiou, "Effect of chemical structure on degree of conversion in light-cured dimethacrylate-based dental resins," Biomaterials, vol. 23, no. 8, pp. 1819-1829, 2002.

[36] J. E. Elliott, L. G. Lovell, and C. N. Bowman, "Primary cyclization in the polymerization of bis-GMA and TEGDMA: a modeling approach to understanding the cure of dental resins," Dental Materials, vol. 17, no. 3, pp. 221-229, 2001.

[37] W. Schroeder, G. Arenas, and C. Vallo, "Monomer conversion in a light-cured dental resin containing 1-phenyl-1,2- propanedione photosensitizer," Polymer International, vol. 56, no. 9, pp. 1099-1105, 2007.

[38] W. F. Schroeder, W. D. Cook, and C. I. Vallo, "Photopolymerization of N,N-dimethylaminobenzyl alcohol as amine co-initiator for light-cured dental resins," Dental Materials, vol. 24, no. 5, pp. 686-693, 2008. 
[39] W. C. Brandt, L. D. O. Tomaselli, L. Correr-Sobrinho, and M. A. C. Sinhoreti, "Can phenyl-propanedione influence Knoop hardness, rate of polymerization and bond strength of resin composite restorations?" Journal of Dentistry, vol. 39, no. 6, pp. 438-447, 2011.

[40] J. Nie and C. N. Bowman, "Synthesis and photopolymerization of N,N/-dimethyl,-N,N/-di(methacryloxy ethyl)-1,6hexanediamine as a polymerizable amine coinitiator for dental restorations," Biomaterials, vol. 23, no. 4, pp. 1221-1226, 2002.

[41] K. Studer and R. Königer, "Initial photoyellowing of photocrosslinked coatings," European Coatings Journal, no. 1-2, pp. 109-110, 2001.

[42] A. Peutzfeldt and E. Asmussen, "Hardness of restorative resins: effect of camphorquinone, amine, and inhibitor," Acta Odontologica Scandinavica, vol. 47, no. 4, pp. 229-231, 1989.

[43] J. W. Stansbury, "Curing dental resins and composites by photopolymerization," Journal of Esthetic Dentistry, vol. 12, no. 6, pp. 300-308, 2000.

[44] M. G. Neumann, W. G. Miranda Jr., C. C. Schmitt, F. A. Rueggeberg, and I. C. Correa, "Molar extinction coefficients and the photon absorption efficiency of dental photoinitiators and light curing units," Journal of Dentistry, vol. 33, no. 6, pp. 525532, 2005.

[45] M. G. Neumann, C. C. Schmitt, I. C. Correa, and B. E. Goi, "The effect of using mixed initiator systems on the efficiency of photopolymerization of dental resins," Journal of the Brazilian Chemical Society, vol. 19, no. 7, pp. 1413-1417, 2008.

[46] R. L. Sakaguchi and H. X. Berge, "Reduced light energy density decreases post-gel contraction while maintaining degree of conversion in composites," Journal of Dentistry, vol. 26, no. 8, pp. 695-700, 1998.

[47] M. S. Soh and A. U. J. Yap, "Influence of curing modes on crosslink density in polymer structures," Journal of Dentistry, vol. 32, no. 4, pp. 321-326, 2004.

[48] E. Asmussen and A. Peutzfeldt, "Influence of pulse-delay curing on softening of polymer structures," Journal of Dental Research, vol. 80, no. 6, pp. 1570-1573, 2001.

[49] M. Väkiparta, M. Puska, and P. K. Vallittu, "Residual monomers and degree of conversion of partially bioresorbable fiberreinforced composite," Acta Biomaterialia, vol. 2, no. 1, pp. 2937, 2006.

[50] H. Müller, S. Olsson, and K.-J. Söderholm, "The effect of comonomer composition, silane heating, and filler type on aqueous TEGDMA leachability in model resin composites," European Journal of Oral Sciences, vol. 105, no. 4, pp. 362-368, 1997. 

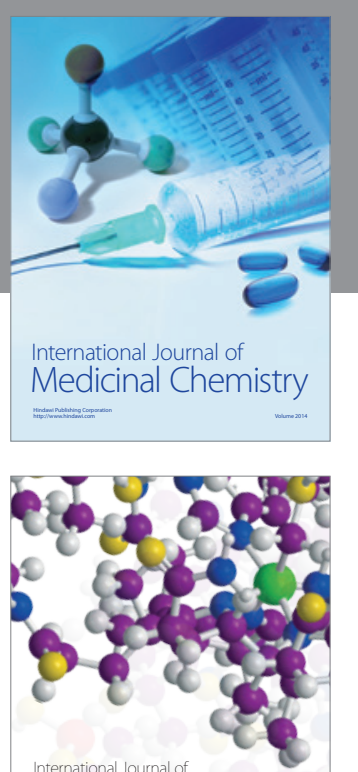

Carbohydrate Chemistry

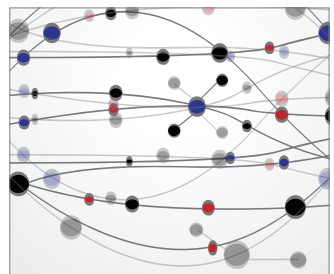

The Scientific World Journal
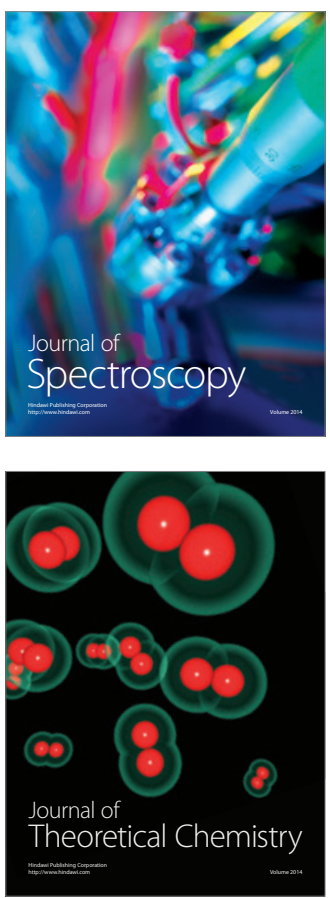
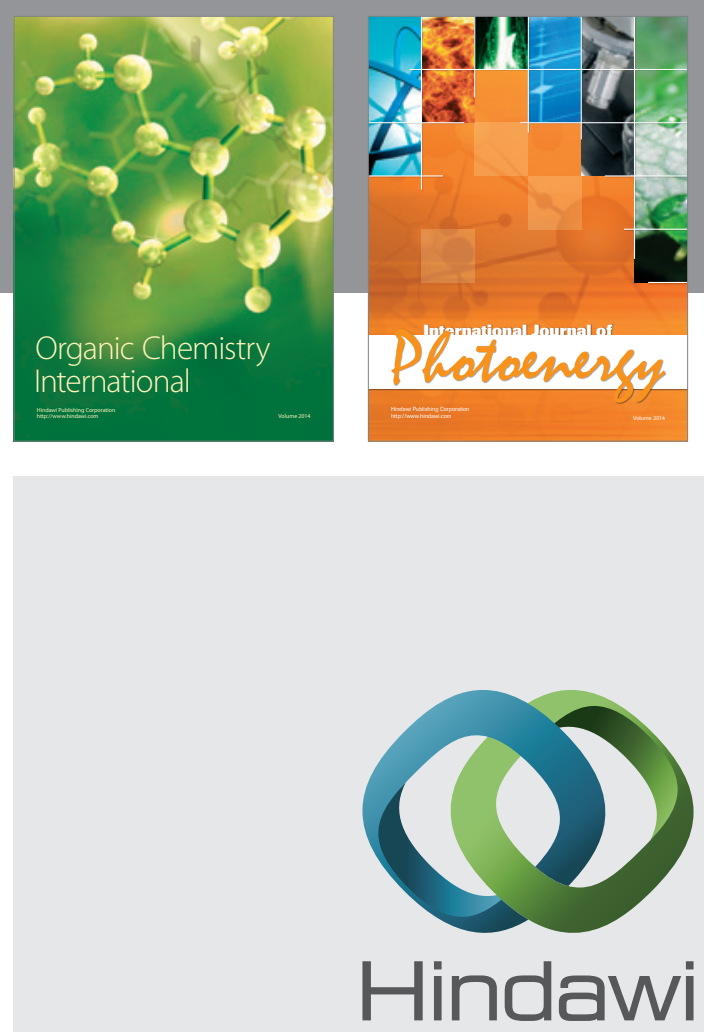

Submit your manuscripts at

http://www.hindawi.com

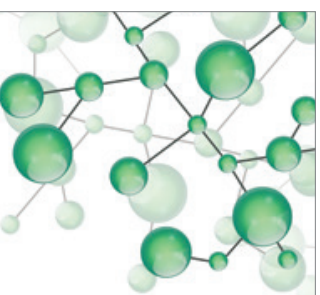

International Journal of

Inorganic Chemistry

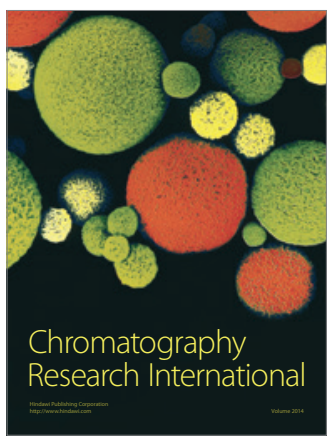

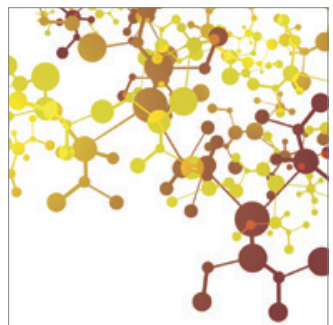

Applied Chemistry
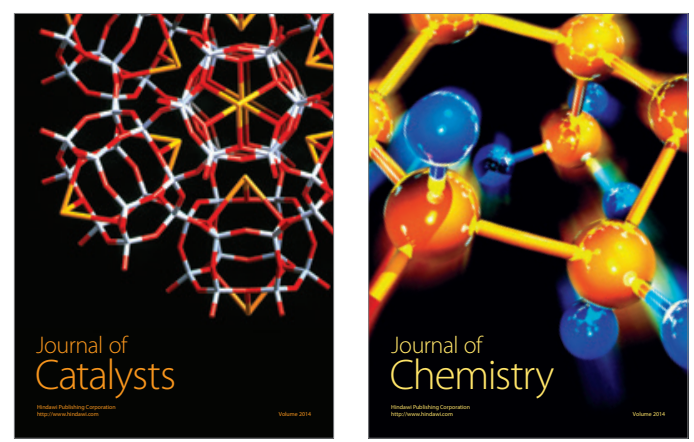
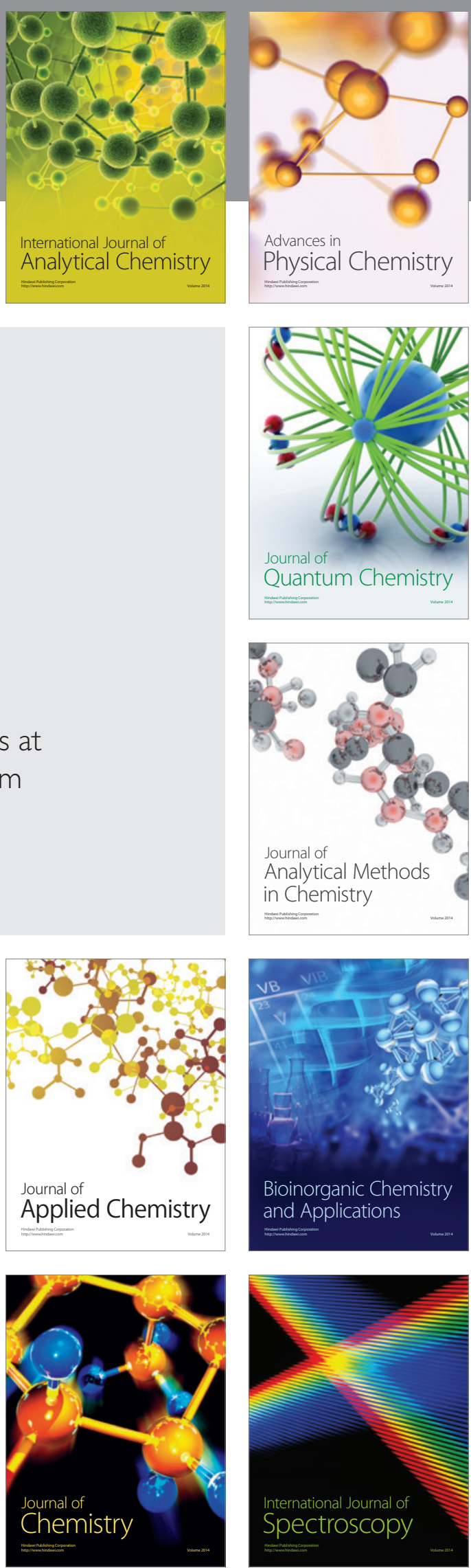